\title{
RESONANCE IN UNDAMPED SECOND-ORDER NONLINEAR EQUATIONS WITH PERIODIC FORCING
}

\author{
BY \\ GEORGE SEIFERT \\ Iowa State University, Ames, Iowa
}

We consider the scalar equation

$$
x^{\prime \prime}+g(x)=p(t)
$$

where $g$ and $p$ are real valued functions continuous on the reals $R, p(t+2 \pi)=p(t)$, and the solutions of (1) are uniquely determined by their initial conditions. If $g$ is nonlinear, the question of whether all solutions of (1) are bounded on $R$ has long been recognized as nontrivial and challenging. For the special case of $g(x)=2 x^{3}$ Morris [1] was able to show that this question has an affirmative answer. Later Dieckerhoff and Zehnder [2] were able to prove it for the case $g(x)=x^{2 n+1}+$ $p_{2 n}(t) x^{2 n}+\cdots+p_{1}(t) x$ where the $p_{k}(t)$ are $2 \pi$-periodic and sufficiently smooth. A more recent and considerably more general result is due to Ding [3] and asserts that if $g$ is continuously differentiable and $g(x) / x \rightarrow \infty$ as $|x| \rightarrow \infty$, the answer is in the affirmative. On the other hand, Littlewood [4] has given an example of an equation like (1) with $g(x) / x \rightarrow \infty$ as $|x| \rightarrow \infty$ where $g(x)$ and $p(t)$ are not continuous which has an unbounded solution on $R$. As Morris has pointed out in [1], it is easy to modify the $g(x)$ in Littlewood's example so that it is continuously differentiable, in fact, $C^{\infty}$, and still have an unbounded solution for (1); whether the function $p(t)$, which in Littlewood's example is piecewise constant-valued, can be modified to be continuous seems not entirely obvious. However, in Ding's proof in [3], only the fact that the Poincare map associated with (1) is an area-preserving twist homeomorphism is used, and since this is also true if $g(x)$ is continuously differentiable and $p(t)$ only piecewise continuous, a question arises as to the validity of Ding's proof or Littlewood's example.

In this note we consider the problem of giving conditions on $g$ for which all solutions of (1) are unbounded on $R$; specifically on $[0, \infty)$, primarily for cases where $g$ is nonlinear. Our main result states that if for some integer $n,\left|g(x)-n^{2} x\right|$ is bounded on $R$, and if a suitable Fourier coefficient of $p(t)$ has sufficiently large absolute value, then all solutions of $(1)$ are unbounded on $[0, \infty)$. 
Our result is based on the following two results, which we state without proofs. First we define for $f(t)$ any function continuous on $[0,2 \pi]$ the notation

$$
M_{f}(n, \delta)=\int_{0}^{2 \pi} f(t) \cos (n t+\delta) d t .
$$

LEMMA 1. If $f(t)$ is continuous and $2 \pi$-periodic, and there exists a $\delta$ such that $M_{f}(n, \delta) \neq 0$, then all solutions of $x^{\prime \prime}+n^{2} x=f(t)$ are unbounded on $[0, \infty)$.

LEMMA 2. If all solutions of (1) exist for $t \geq 0$ and there exists a solution which together with its derivative is bounded on $[0, \infty)$, then there exists a $2 \pi$-periodic solution of (1).

Lemma 1 is well known and easy to prove; in fact it also follows easily that if $M_{f}(n, \delta)=0$ for all $\delta, 0 \leq \delta<2 \pi$, then all solutions of $x^{\prime \prime}+n^{2} x=f(t)$ are bounded on $R$. Lemma 2 is a special case of a theorem due to Massera [5] and its proof uses a nontrivial fixed point theorem in $R^{2}$.

THEOREM 1. Suppose there exists a $g_{0}>0$, and an integer $n \geq 0$, such that

$$
\left|g(x)-n^{2} x\right| \leq g_{0} \text { for } x \text { in } R
$$

Then if $\left|M_{p}\left(\delta_{0}, n\right)\right|>2 \pi g_{0}$, where $\delta_{0}=-\tan ^{-1}\left(s_{0} / c_{0}\right), c_{0}=\int_{0}^{2 \pi} p(t) \cos n t d t$, $s_{0}=\int_{0}^{2 \pi} p(t) \sin n t d t$, all solutions of $(1)$ are unbounded on $[0, \infty)$.

Proof. Using standard continuation theorems, one can easily show that under the hypotheses on $g$, all solutions of (1) can be continued to $t=\infty$; we omit the details.

We next show that if $x(t)$ is a solution bounded on $[0, \infty)$ then $x^{\prime}(t)$ is also. Suppose not; then there exists $t_{0}>0$ such that

$$
\left(x^{\prime}\left(t_{0}\right)\right)^{2}>4 M_{0} B_{0}
$$

where $B_{0}=\sup \{|x(t)|: t \geq 0\}$, and $M_{0}=\sup \left\{|g(x)-p(t)|:|x| \leq B_{0}, t\right.$ in $\left.R\right\}$.

Suppose $x^{\prime}\left(t_{0}\right)>0$; the case $x^{\prime}\left(t_{0}\right)<0$ can be dealt with in the same way. Since $x(t)$ increases without bound if $x^{\prime}(t)>0$ and is bounded away from zero for $t \geq 0$, it follows that there exists an increasing sequence $\left\{t_{k}\right\}, k=1,2, \ldots$, such that $t_{1}>t_{0}, x^{\prime}\left(t_{k}\right) \rightarrow 0$ as $k \rightarrow \infty$, and $x^{\prime}(t)>0$ for $t_{0} \leq t<\bar{t}$ where $\bar{t}=\lim _{k \rightarrow \infty} t_{k}$. Put $x_{k}=x\left(t_{k}\right), k=0,1,2, \ldots$, and define $y(x)=x^{\prime}(t(x))$, where $t(x)$ is the inverse of $x(t)$ on $\left[t_{0}, \bar{t}\right)$. It is easy to show that for any integer $k \geq 1, d\left(y^{2}(x)\right) / d x=2(p(t(x))-g(x)), x_{0}<x<x_{k}$.

From this and the mean value theorem we get

$$
\left|y^{2}\left(x_{k}\right)-y^{2}\left(x_{0}\right)\right| \leq 2 M_{0}\left(x_{k}-x_{0}\right) \leq 4 M_{0} B_{0}
$$

and since $y\left(x_{0}\right)=x^{\prime}\left(t_{0}\right)$ and $y\left(x_{k}\right)=x^{\prime}\left(t_{k}\right) \rightarrow 0$ as $k \rightarrow \infty$, we obtain from (2) that $\left(x^{\prime}\left(t_{0}\right)\right)^{2} \leq 4 M_{0} B_{0}$, which contradicts (1.2). This proves the assertion that $x^{\prime}(t)$ is alsobounded on $[0, \infty)$. 
Thus if (1) has a solution bounded on $[0, \infty)$, by Lemma 2 it has a $2 \pi$-periodic solution, say $\bar{x}(t)$. So the equation

$$
x^{\prime \prime}+n^{2} x=n^{2} \bar{x}(t)-g(\bar{x}(t))+p(t)
$$

has a $2 \pi$-periodic solution $\bar{x}(t)$. Using Lemma 1 with $f(t)=n^{2} \bar{x}(t)-g(\bar{x}(t))+p(t)$ we find that for all $\delta$,

$$
M_{p}(\delta, n)=-\int_{0}^{2 \pi}\left(n^{2} \bar{x}(t)-g(\bar{x}(t)) \cos (n t+\delta) d t .\right.
$$

So $\left|M_{p}(\delta, n)\right| \leq 2 \pi g_{0}$ for all $\delta$. But by elementary calculus, it follows easily that

$$
\max \left\{M_{p}(\delta, n): \delta \text { real }\right\}=\left|M_{p}\left(\delta_{0}, n\right)\right|,
$$

and we arrive at a contradiction since by hypotheses $\left|M_{p}\left(\delta_{0}, n\right)\right|>2 \pi g_{0}$. This proves the theorem.

We state a result for a more general equation which can be proved using the method in the proof of Theorem 1 .

THEOREM 2. Let $f(x, t)$ be real valued and continuous of $R \times R$ and $f(x, t+2 \pi)=$ $f(x, t)$ there. Suppose there exists an integer $n>0$ and a $\delta$ such that

$$
\int_{0}^{2 \pi}\left(f(\bar{x}(t), t)+n^{2} \bar{x}(t)\right) \cos (n t+\delta) d t \neq 0
$$

for all continuously differentiable $2 \pi$-periodic functions $\bar{x}(t)$. Suppose all solutions of

$$
x^{\prime \prime}=f(t, x)
$$

are uniquely determined by these initial conditions and extend to $[0, \infty)$. Then all solutions of (4) are unbounded on $[0, \infty)$.

We conclude by applying Theorem 1 to some special cases of (1), first to obtain a lower bound on the suprema of the absolute values of certain bounded solutions of $x^{\prime \prime}+x^{3}=p(t)$.

Let $n_{0}$ and $m_{0}$ be positive integers, and define

$$
\begin{aligned}
g(x) & =x^{3}, \quad 0 \leq x \leq m_{0}, \\
& =n_{0}^{2}\left(x-m_{0}\right)+m_{0}^{3}, x_{0}>m_{0}
\end{aligned}
$$

and $g(x)=-g(-x)$ for $x<0$. We use Theorem 1 with $n=n_{0}$. If

$$
g_{0}=\max \left\{m_{0}\left|m_{0}^{2}-n_{0}^{2}\right|, 2 n_{0}^{3} / 3 \sqrt{3}\right\},
$$

it follows by straightforward calculations that

$$
\left|g(x)-n_{0}^{2} x\right| \leq g_{0}, x \text { in } R
$$

Thus if $M_{p}\left(n_{0}, \delta_{0}\right)>2 \pi g_{0}$, with $\delta_{0}$ as defined in Theorem 1 with $n=n_{0}$, all solutions of $(1)$ for this $g$ are unbounded on $[0, \infty)$; i.e., for any solution $x(t)$ of (1) there exist $t_{k} \rightarrow \infty$ as $k \rightarrow \infty$ such that $\left|x\left(t_{k}\right)\right|>m_{0}, n=1,2, \ldots$. 
This shows also that if $x(t)$ solves $x^{\prime \prime}+x^{3}=p(t)$ and $|x(t)| \leq m_{0}$ for $t$ in $R$, then $M_{p}\left(n_{0}, \delta_{0}\right) \leq 2 \pi g_{0}$.

If we take $m_{0}=n_{0}$, clearly $g_{0}=2 n_{0}^{3} / 3 \sqrt{3}$, and we conclude that if

$$
\int_{0}^{2 \pi} p(t) \cos \left(n_{0} t+\delta_{0}\right) d t>4 \pi n_{0}^{3} / 3 \sqrt{3}
$$

then there exist no solutions $x(t)$ of $x^{\prime \prime}+x^{3}=p(t)$ such that $|x(t)| \leq n_{0}$ for $t$ in $R$.

Next consider the piecewise linear $g(x)$ given by

$$
\begin{aligned}
g(x) & =x, & & 0 \leq x \leq 1, \\
& =4 x-3, & & x>1
\end{aligned}
$$

with $g(x)=-g(-x), x<0$.

A simple application of Theorem 1 shows that if $\delta_{0}$ is defined as in Theorem 1 with $n=2$, and

$$
\left|M_{p}\left(2, \delta_{0}\right)\right|>6 \pi
$$

then all solutions of (1) are unbounded on $[0, \infty)$. An interesting and apparently open question is that if $p(t)$ does not satisfy (5), are these solutions bounded on $[0, \infty)$; i.e., are there $2 \pi$-periodic solutions? It is easy to show that if for some $\delta$,

$$
M_{p}(1, \delta) \neq 0,
$$

then for every solution $x(t)$ there exists a sequence $t_{k} \rightarrow \infty$ as $k \rightarrow \infty$ such that $\left|x\left(t_{k}\right)\right|>1, k=1,2, \ldots$. If not, there exists a $t_{0}$ such that $|x(t)| \leq 1$ for $t \geq t_{0}$, and so $x(t)$ satisfies $x^{\prime \prime}+x=p(t)$ for all such $t$, and using (6) we easily get a contradiction.

\section{REFERENCES}

[1] G. Morris, A case of boundedness in Littlewood's problem on oscillatory differential equations, Bull. Austral. Math. Soc. 14, 71-93 (1976)

[2] R. Dieckerhoff and R. Zehnder, Boundedness of solutions via twist theorem. Abteilung für Math. der Ruhr-Univ., Bochum 22 (1984)

[3] T. Ding, An answer to Littlewood's problem on houndedness for super-linear Duffing's equations, J. Diff. Equations 73 (2), 269-287 (1988)

[4] J. E. Littlewood, Lnbounded solutions of an equation $\ddot{y}+g(y)=p(t)$, with $p(t)$ periodic and bounded and $g(y) / y \rightarrow \infty$ as $y \rightarrow \pm \infty$, J. London Math. Soc. 41. 497-507 (1966)

[5] J. Massera, The existence of periodic solutions of sistems of differential equations. Duke Math. J. 17, 457-475 (1950) 